\title{
Impact of rigorous clinical and laboratory screening for coronavirus disease 2019 (COVID-19) in a teaching hospital in inner Brazil
}

\author{
Stephanie Valentini Ferreira Proença MD¹ , Carlos Magno Castelo Branco Fortaleza PhD ${ }^{1,2}$ (1) , Jonas Atique Sawazaki MS ${ }^{1,2}$, \\ Ricardo de Souza Cavalcante $\mathrm{PhD}^{1,2}$ (1) Bruno Cardoso de Macedo MD ${ }^{1,2}$, Sandra Mara Queiroz $\mathrm{MD}^{2}$, \\ Sebastião Pires Ferreira Filho $\mathrm{MS}^{2}$ and Gabriel Berg de Almeida $\mathrm{MS}^{1,2}$ (1) \\ ${ }^{1}$ Infectious Diseases Department, Botucatu Medical School, São Paulo State University (UNESP), Botucatu, São Paulo, Brazil and ${ }^{2}$ Clinical Hospital of Botucatu \\ Medical School, Botucatu, São Paulo, Brazil
}

To the Editor-The greater spread of severe acute respiratory coronavirus virus 2 (SARS-CoV-2) variants of concern (VOCs) determines the occurrence of community outbreaks and quickly poses a potential risk for nosocomial outbreaks. ${ }^{1,2}$ Given the estimated basic reproduction number of the virus, the transmission through respiratory droplets, the possibility of transmission of asymptomatic and oligosymptomatic individuals, and the high occupancy rate of the health services, the hospital environment has become a risk scenario, especially susceptible to outbreaks and compromising patients with potential for unfavorable clinical outcomes. ${ }^{3}$ In February 2022 in Brazil, official data from the Ministry of Health estimated $>24,000,000$ confirmed cases of COVID-19, with $>620,000$ deaths. The incidence of transmission of SARS-CoV-2 within hospital settings is unknown. Few hospitals have reported healthcare-associated coronavirus disease 2019 (COVID-19) cases to the Brazilian Healthcare-Associated Infections Program, with rates varying from $0.23 \%$ to $2.5 \%$ of all admissions. ${ }^{4}$ Policies for identifying COVID-19 among those admitted for other reasons is of utmost importance for preventing outbreaks.

In March 2021, we implemented a clinical and laboratory screening strategy in the emergency unit and hospital wards of the Clinical Hospital of Botucatu Medical School. Our hospital has 500 beds in wards, including 53 in intensive care units and an emergency room (ER). It is the referral facility for tertiary care for an area comprising $>500,000$ inhabitants in inner São Paulo State, Brazil (22053'09”S, $48^{\circ} 26^{\prime} 42^{\prime \prime} \mathrm{W}$ ). The $\gamma$ (gamma) variant was dominant at the time, representing up to $90 \%$ of the sequenced samples in Brazil. ${ }^{5}$

In addition to the standard measures of infection control (exclusive wards for COVID-19, restriction of visits and patients' companions, universal use of masks, social distancing measures), a daily checklist of flu-like symptoms was applied throughout the hospital stay for every patient. Those presenting any symptom had the appropriate precautions immediately instituted and were tested for SARS-CoV-2 through real-time polymerase chain reaction (RT-PCR). Additionally, RT-PCR in pooled saliva was performed for all patients on the day of admission.

A similar strategy was used for healthcare workers (HCWs), with daily screening for symptoms and weekly collection of saliva pools for those at high occupational risk. HCWs could report symptoms via mobile phone or through the electronic hospital chart.

From March through October 2021, we identified 25 COVID-19 patients through symptom screening and RT-PCR. The saliva-pool

\footnotetext{
Author for correspondence: Gabriel Berg de Almeida, E-mail: gb.almeida@unesp.br Cite this article: Proença SVF, et al. (2022). Impact of rigorous clinical and laboratory screening for coronavirus disease 2019 (COVID-19) in a teaching hospital in inner Brazil. Infection Control \& Hospital Epidemiology, 43: 1970-1971, https://doi.org/10.1017/ ice.2022.59
}

strategy identified 13 additional asymptomatic SARS-CoV-2 infections. Moreover, 134 contacts (ie, patients in the same room as index case) were investigated, both in the ER and in the inpatient wards.

Of the 25 patients identified in symptoms screening, 19 produced no secondary cases, 1 patient transmitted to 1 contact patient each and 1 patient transmitted to 3 contact patients. We also identified 1 case of superspreading to 7 secondary patients. Among the asymptomatic individuals detected through saliva screening, 9 produced no secondary cases and 4 transmitted to 1 contact each. Therefore, the secondary case rate was 0.56 (standard error $[\mathrm{SE}] \pm 0.30$ ) for symptomatic source patients and 0.31 ( $\mathrm{SE} \pm 0.13$ ) for asymptomatic source patients, a difference that did not reach statistical significance $(P=.56)$.

We performed a single-step Poisson regression model including source patient classification (symptomatic vs asymptomatic) and hospital site where they were admitted (ER vs wards). Results pointed to the ER as a site with greater risk for nosocomial transmission (rate ratio $[\mathrm{RR}], 2.72$; 95\% confidence interval [CI], 1.05-7.04; $P<.05)$ but no transmission advantage among symptomatic sources (RR, 1.83; 95\% CI, $0.61-5.67 ; P=.27)$.

Due to the scarcity of hospital beds in the peak $\gamma$ (gamma) variant spread, some patients stayed in the ER for several days. Those who were not admitted with suspected COVID-19 were frequently grouped in large rooms with $>50$ beds. It is, therefore, no surprise that nosocomial SARS-CoV-2 transmission occurred in the ER.

On the other hand, the number of secondary cases was equally low (and $<1$ per source) for those detected by symptom screening or by pooled -saliva RT-PCR. We firmly believe that rapid identification and contact tracing were essential for preventing outbreaks.

Our findings are consistent with the literature, which confirms the possibility and reports the occurrence of in-hospital transmission of COVID-19, and suggests that control measures are necessary. ${ }^{6}$ A study from the United Kingdom estimated rates of occurrence of in-hospital COVID-19 at up to $15 \%$ of all cases admitted after the peak admission period. ${ }^{7}$ In contrast, a cohort study in a large US hospital showed that only $1.7 \%$ of patients admitted in 12 weeks had nosocomial COVID-19, demonstrating that strict hospital control makes the transmission of SARS-CoV-2 in their environment rare. ${ }^{8}$

At the peak of COVID-19 deaths in Brazil, infection control policies were required to prevent the collapse of hospitals, which frequently included a shortage of beds and oxygen. ${ }^{9}$ By preventing nosocomial outbreaks, our strategy was partially responsible for keeping the hospital at its total capacity. In conclusion, strict hospital infection control may be associated with lower SARS-CoV-2 transmission in hospitalized patients.

\section{Acknowledgments.}

Financial support. No financial support was provided relevant to this article. 
Conflicts of interest. All authors report no conflicts of interest relevant to this article.

\section{References}

1. Shitrit $\mathrm{P}$, Zuckerman NS, Mor O, et al. Nosocomial outbreak caused by the SARS-CoV-2 delta variant in a highly vaccinated population, Israel, July 2021. Euro Surveill 2021;26:2100822.

2. Lim WY, Tan GSE, Htun HL, et al. First nosocomial cluster of COVID-19 due to the delta variant in a major acute-care hospital in Singapore: investigations and outbreak response. J Hosp Infect 2021;122:27-34.

3. Abbas M, Robalo Nunes T, Martischang R, et al. Nosocomial transmission and outbreaks of coronavirus disease 2019: the need to protect both patients and healthcare workers. Antimicrob Resist Infect Control 2021;10:7.

4. Divulgado boletim Informativo sobre transmissão de COVID-19 hospitalar. Agência Nacional de Vigilância Sanitária (ANVISA) website. https:// www.gov.br/anvisa/pt-br/assuntos/noticias-anvisa/2021/divulgado-boletim- sobre-transmissao-hospitalar-da-covid-19. Published 2022. Accessed February 12, 2022.

5. Dashboard Rede Genômica-Vigilância Genômica do SARS-CoV-2 no Brasil. Fundação Osvaldo Cruz (Fiocruz) website. http://www.genomahcov.fiocruz.br/ dashboard/ Published 2022. Accessed February 12, 2022.

6. Pham TM, Tahir H, van de Wijgert JHHM, et al. Interventions to control nosocomial transmission of SARS-CoV-2: a modelling study. BMC Med 2021;19:211.

7. Read JM, Green CA, Harrison EM, et al. Hospital-acquired SARS-CoV-2 infection in the UK's first COVID-19 pandemic wave. Lancet 2021;398:1037-1038.

8. Rhee C, Baker M, Vaidya V, et al. Incidence of nosocomial COVID-19 in patients hospitalized at a large US academic medical center. JAMA Netw Open 2020;3(9):e2020498.

9. Zeiser FA, Donida B, da Costa CA, et al. First and second COVID-19 waves in Brazil: a cross-sectional study of patients' characteristics related to hospitalization and in-hospital mortality. Lancet Reg Health Am 2022;6:100107.

\title{
False-negative nasopharyngeal severe acute respiratory coronavirus virus 2 (SARS-CoV-2) reverse-transcription polymerase chain reaction (RT-PCR) in immunocompromised patients resulting in healthcare worker exposures
}

\author{
Elena Beam MD ${ }^{1,2}$ (1) , Michelle L. Meyer PhD², John C. O' Horo MD, MPH ${ }^{1,2,3}$ and Laura E. Breeher MD, MPH ${ }^{4,5}$ \\ ${ }^{1}$ Division of Infectious Diseases, Mayo Clinic, Rochester, Minnesota, ${ }^{2}$ Infection Prevention and Control, Mayo Clinic, Rochester, Minnesota, ${ }^{3}$ Division of Pulmonary \\ and Critical Care Medicine, Mayo Clinic, Rochester, Minnesota, ${ }^{4}$ Division of Preventive, Occupational, and Aerospace Medicine, Rochester, Minnesota and \\ ${ }^{5}$ Occupational Health Services, Rochester, Minnesota
}

To the Editor-Exposure to coronavirus disease 2019 (COVID-19) infection will remain a concern in healthcare settings, even with increasing vaccination rates among healthcare staff and patient populations. This is particularly true for healthcare workers (HCWs) who are immunocompromised because they have been noted to have a lower response to available COVID-19 vaccines. ${ }^{1-6}$ Specific immunocompromised hosts, including hypogamma-globulinemic patients and those on anti-CD-20 inhibitors, are not only at risk for poor vaccine response but can also present with prolonged duration of symptoms and infection.

We describe 2 immunocompromised patients who were noted to have negative severe illness and acute respiratory coronavirus virus 2 (SARS-CoV-2) testing on nasopharyngeal (NP) swabs. Both patients were admitted for abnormal computed tomography chest findings, with associated respiratory symptoms of 42 - and 100-day durations, respectively. Both cases occurred in immunocompromised individuals: a male aged 57 years with mantle cell lymphoma on rituximab and a female aged 59 years with history of follicular lymphoma on obinutuzumab therapy. Isolation precautions were discontinued once NP swab results returned negative. Both patients subsequently underwent bronchoscopy for bronchoalveolar lavage (BAL) sampling, and SARS-CoV-2 reverse-

Author for correspondence: Elena Beam, E-mail: beam.elena@mayo.edu

Cite this article: Beam E, et al. (2022). False-negative nasopharyngeal severe acute respiratory coronavirus virus 2 (SARS-CoV-2) reverse-transcription polymerase chain reaction (RT-PCR) in immunocompromised patients resulting in healthcare worker exposures. Infection Control \& Hospital Epidemiology, 43: 1971-1972, https://doi.org/ $10.1017 /$ ice. 2021.447 transcriptase polymerase chain reaction (RT-PCR) tests returned positive: one on day 26 of admission (cycle threshold value [Ct], 20.8 ), and the other on day 3 of admission (Ct, 31.8). Both patients received COVID-19-directed therapy with reported symptom improvement.

These 2 cases resulted in large exposure follow up investigations. In the first case, 10 HCWs were evaluated and 2 met significant-risk exposure criteria, both due to lack of eye protection in addition to a face mask when in close, prolonged contact with the unmasked patient [ $<2 \mathrm{~m}$ ( 6 feet) for $>15$ minutes]. The exposed HCWs were both fully vaccinated (complete vaccination series plus 2 weeks) and were offered RT-PCR testing at baseline and days 5-7 following the exposure according to the institution's occupational health and safety recommendations. Neither case resulted in known patient exposure.

In the second case, the delay in diagnosis and use of high-flow oxygen therapy and an Aerobika breathing device throughout prolonged hospitalization resulted in an even larger exposure follow up. In total, $184 \mathrm{HCWs}$ were reviewed for exposures, and 83 were identified as having significant-risk exposures going back 14 days prior to the positive test. Significant risk exposures were due to lack of eye protection when interacting with the unmasked patient and/ or use of a face mask rather than a respirator during an aerosolgenerating procedure or the postprocedure room clearance. Of the 83 exposed HCWs, 70 were fully vaccinated and were offered testing at baseline and day 5-7 following the exposure. Among these 83 HCWs, 13 (16\%) were unvaccinated or were incompletely vaccinated, and serial PCR testing was arranged at baseline, day $5-7$, and days 12-14 following the last exposure. HCWs who were 